\title{
Sky observation and mythology: paths to an astronomical culture
}

\author{
Victor Alves Alencar ${ }^{1,2}$ \\ ${ }^{1}$ Departamento de Física, Centro de Ciencias, \\ Universidade Federal do Ceara, Campus do Pici, \\ 60455-760 Fortaleza, Brazil \\ email: victoralencar@fisica.ufc.br \\ ${ }^{2}$ Scientific and Didactic committee of the Brazilian Astronomy Olympiad, Brazil
}

\begin{abstract}
The teaching and the popularization of Astronomy, nowadays, suffers from the absence of attractiveness. It's, somehow, consensual that the most efficient approach to attract the general public or even an uninterested student to the Astronomy is the sky observation. With the help of instruments like telescopes or binoculars, the observation of aesthetical impressive objects such as galactic clusters, approaching planets and many others may have a great impact upon those people. We have been executing an efficient and attractive didactic method which has been evaluated permanently for more than two years and has shown great efficiency that consists in the utilization of legends and myths from different cultures (including Brazilian Indians myths, in an etnoastronomical approach) about the constellations and their dispositions in the sky. The objective of this panel is to show the efficiency of the method and some of its teaching routines always beginning with myths or legends involving a great number of constellations (using, preferentially, most of the watchable sky in a certain time of year), using asterisms for identification and then following to a certain constellation from which it is possible to extract the information required for the studied subject.
\end{abstract}

Keywords. constellations, popularisation, teaching, didactic methods, etnoastronomy, mythology, sky observations

\section{Introduction}

Astronomy, in the shape of science as we know, came from the direct sky observation. Since the primordial times, men already knew how important the sky was in their daily life - as we can see analyzing the registers of ancient civilisations and the prestige enjoyed by the priests: astronomers who possessed and manipulated this knowledge, used mainly for religious purposes and also for the production of calendars and in agronomy. Nowadays, the astronomic science suffers from a lack of interest, since it is considered a complex science and without practical applications. It is placed on the back burner along with its teaching and its promotion. Reversing this situation is a hard work, which is faced by teachers and diffusers, day after day. But sometimes this goal is not accomplished, for the public has no motivation for it and hence there is no way to transmit the content, producing a low efficiency learning.

In general, the most common strategy employed by astronomy instructors is the approach of direct sky observation (naked eye) or the use of instruments (telescopes and binoculars), aiming at a sky object that has a particularity or that exerts an æesthetical attraction as galactic clusters, planets, comets, among others. This method is efficient at certain degree, but the knowledge does not remain in the spectators mind, and if this is not stimulated, it will disappear. 


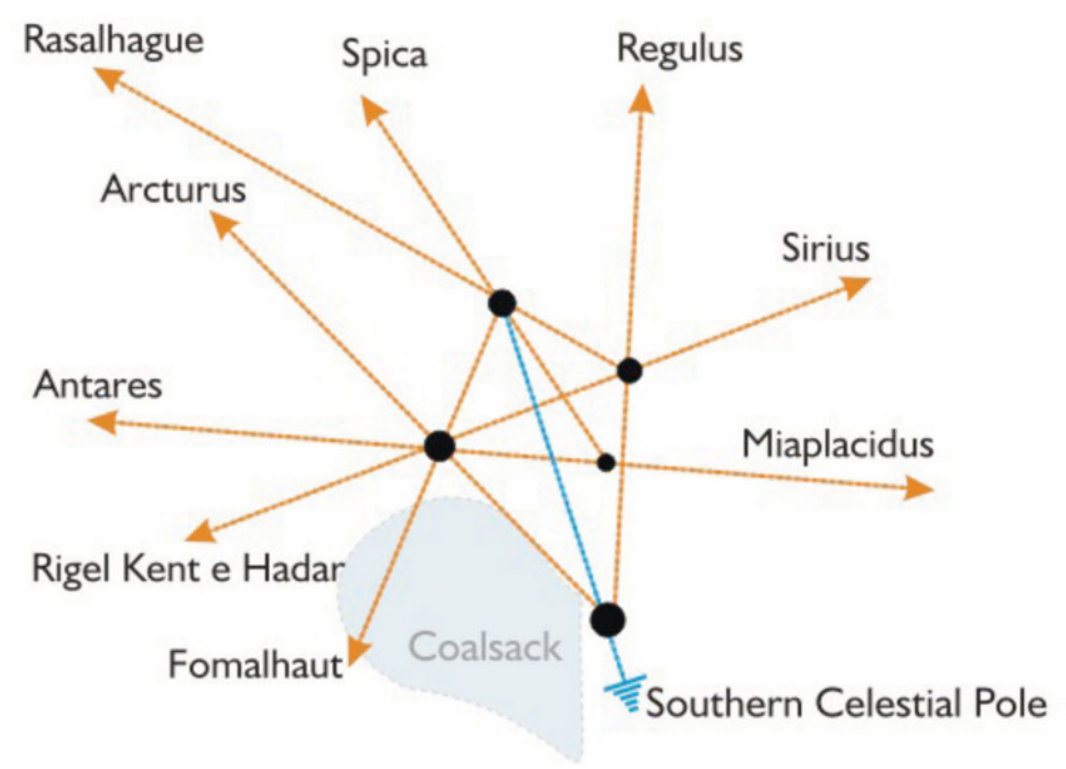

Figure 1. Alignments starting from Crux.

We noticed that, during the explanations about the sky, when we made a mythological reference related to a constellation, the public paid more attention. There was a greater interest in the subject and the flow of questions about the several aspects of the constellation increased. When we put an uncommon legend in the explanation, such as legends about indigenous or ancient peoples, the attention increased, which motivated us to improve this method.

During two years, we tested a teaching method that consists in the use of an interdisciplinary approach, mixing history, mythology and sky observation, with students in different age groups and social levels. The efficiency of this method increased when we noticed the possibility of introducing the most different areas of astronomical knowledge, ranging from the Archeoastronomy to the Extragalactic Astronomy and Cosmology. Considering the importance of the diffusion of the astronomical knowledge and its role in society and culture, this poster aims to show ways to reach a greater degree of efficiency in an observational class. It also contains some suggestions of tested and well successful observation routines that can be used in the assimilation of the explained subject, during the empirical usage of this method.

\section{Methodology}

\subsection{Public}

The method was tested with students of different social levels, in age groups that ranged from 10 to 19 years old, focusing the students of public and private schools between 14 and 19 years old (which were more numerous), in local, regional and national scales. In this sample, it is possible to observe the most different levels of astronomical knowledge (from nave students to students selected to the eliminatory phases of the International Astronomy Olympiads - IAO and IOAA). 


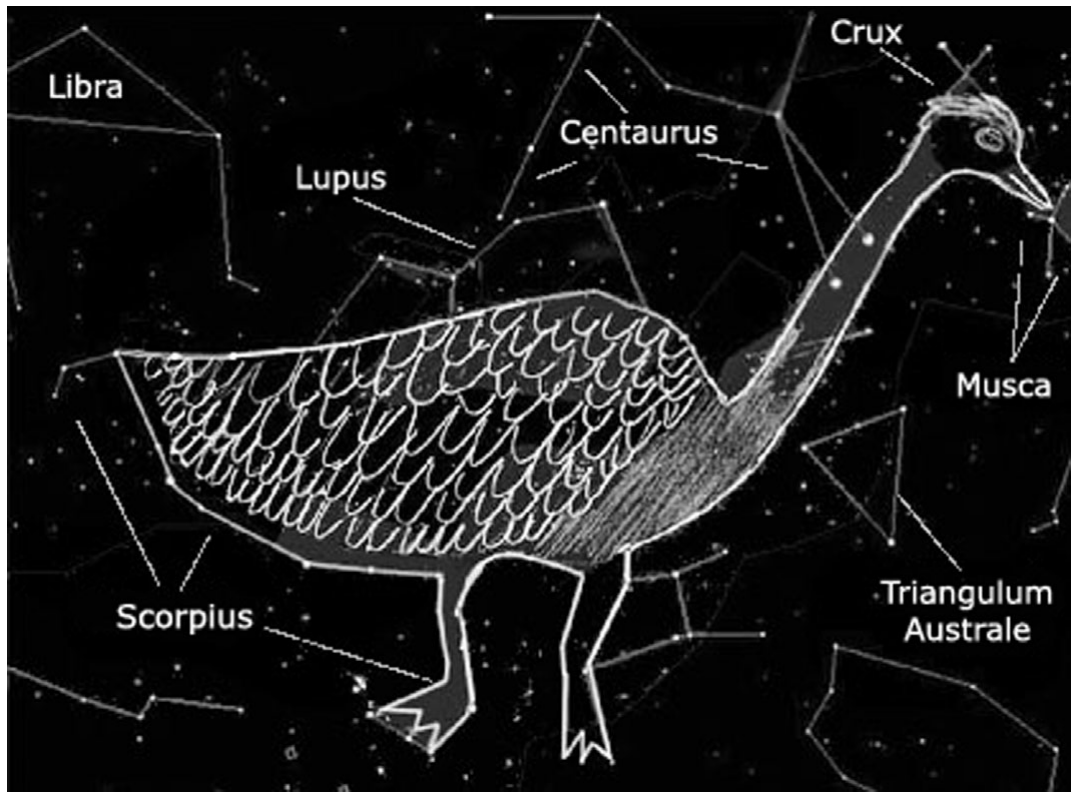

Figure 2. Celestial Rhea.

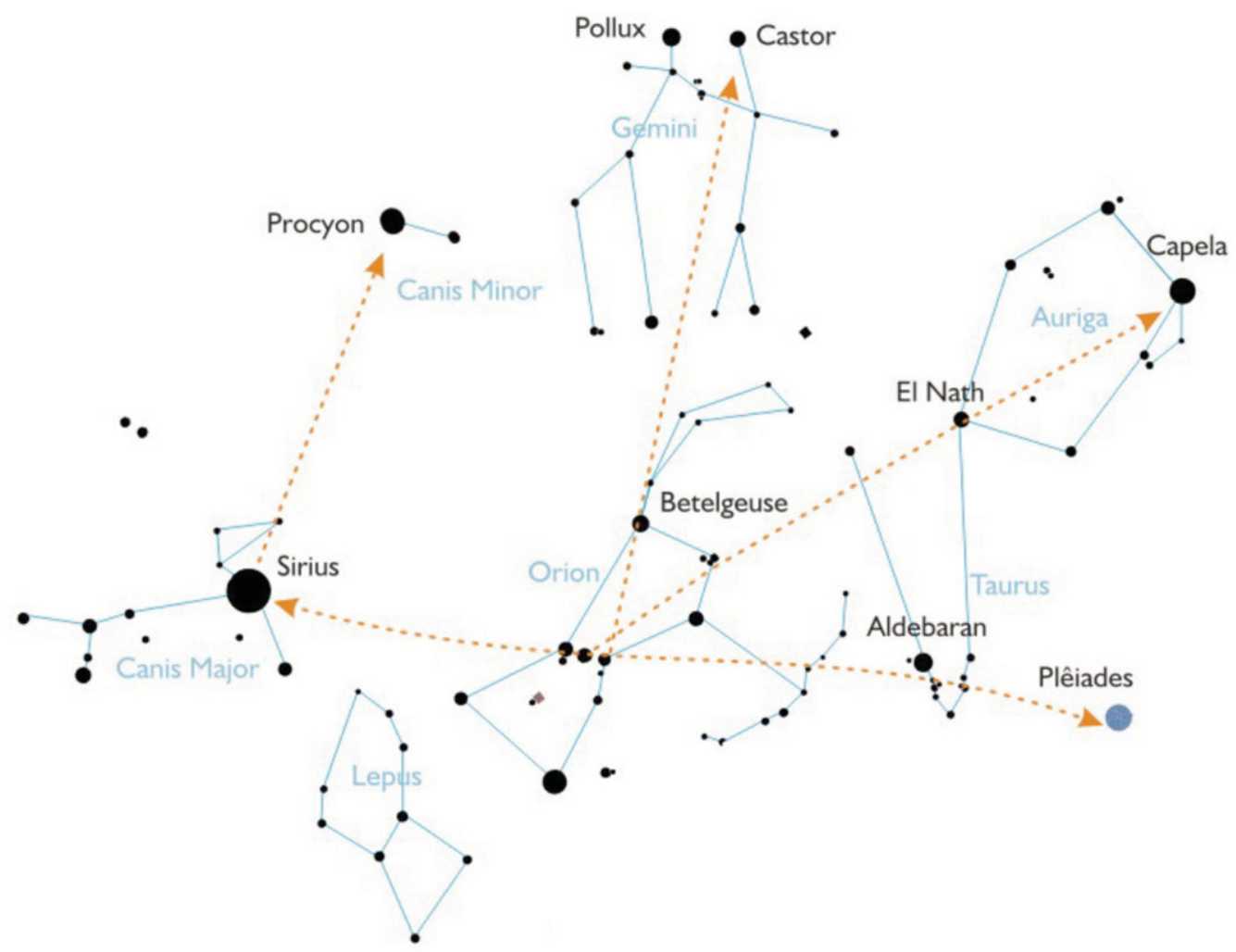

Figure 3. Alignments starting from Orion 


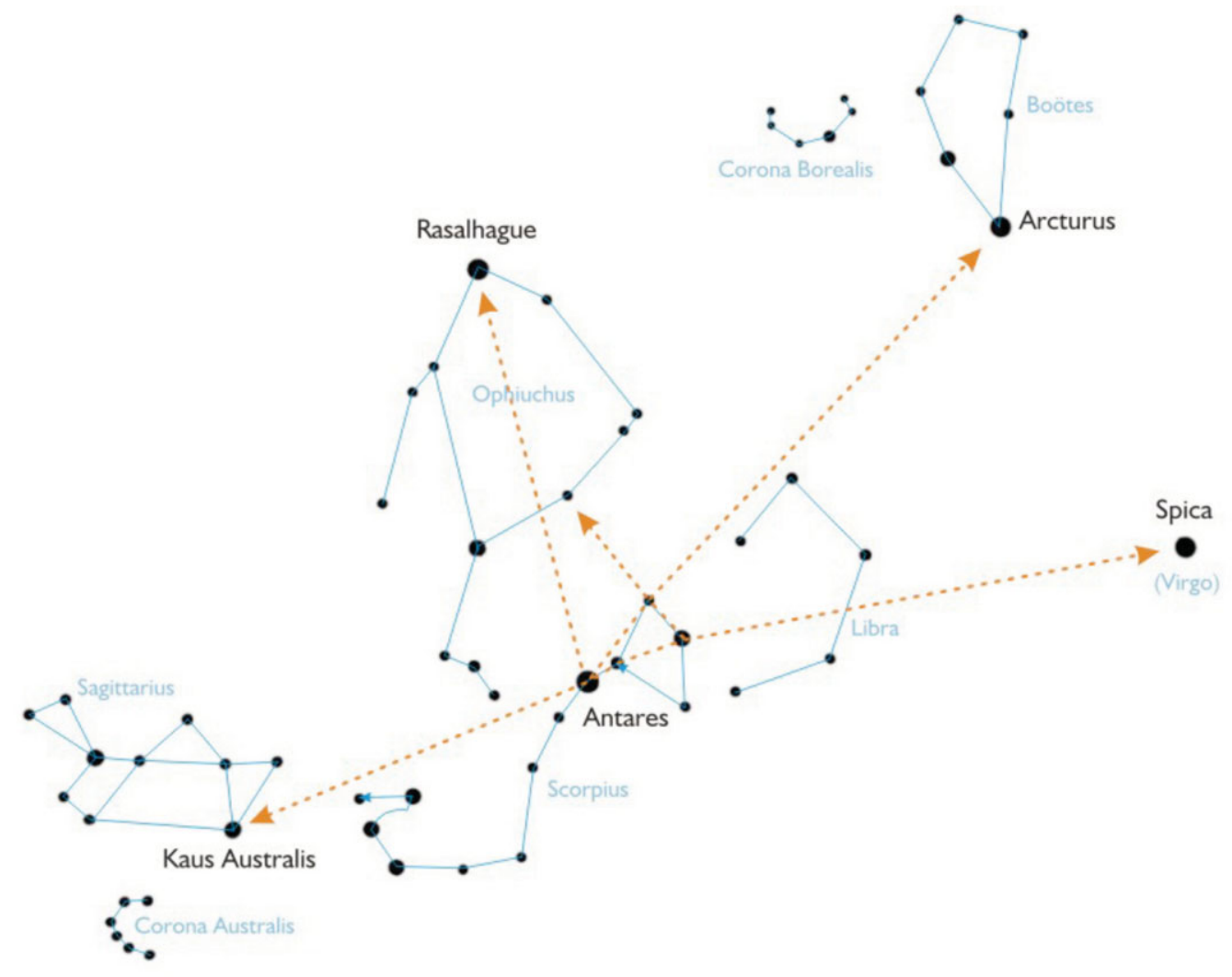

Figure 4. Alignments starting from Scorpius.

\subsection{Application and Period}

The testing period of this teaching methodology lasted nearly two years and it was applied in different formats: curricular classes (geography and physics), field classes, lectures and workshops.

\subsection{Method}

The method consists in the use of history and mythology of several cultures related to constellations, stars, clusters, among others. The legend that will be used must, preferentially, encompass a significant portion of the sky. In this portion, there must be mnemonic alignments in order to consolidate the sky as a whole and to develop the knowledge of other disciplines desired by the teacher. Thus, several routines were created involving constellations with these mnemonic alignments and the related mythology. The mythology that will be approached is chosen in accordance with the teacher, but the use of unknown legends for most of people (or the approach of a known legend with new aspects) makes the subject more interesting, as well as the assimilation.

\section{Teaching Routines}

The most different routines involving visible constellations were used, between the latitudes $-30^{\circ}$ and $0^{\circ}$. However, some routines did not attract the public's attention. So, we selected some of the most welcomed routines whose explanation is sequenced, leaving the knowledge more consolidated. The first step is to use a legend in which we 
can identify the most popular constellations in an isolated way. Thus, we selected Orion, Scorpius and Crux. In many civilizations, these constellations played an important role, such as the Southern Cross to the peoples of the Southern Hemisphere. As examples of constellations that can be used in boreal altitudes, there are the Ursa Major and Ursa Minor that are present in many legends.

\subsection{Crux}

This constellation, which was invented in the period of the Great Navigations, does not have an equivalent classic mythology available, but it is mentioned in indian mythology of many peoples in the Southern Hemisphere. Its advantage, due to the fact it is an excellent asterism, is that this constellation is easily recognized and if we link its stars one another, we have several stars from different constellations as a target. This makes possible to expand the knowledge of the sky in this region. Besides, using this constellation, we can easily locate the Celestial South Pole and thus find out the latitude of the place. To some brazilian indigenous ethnical groups, this constellation symbolizes the Mutum (a typical Brazilian bird with black feathers). When the Southern Cross appears in the night sky, this represents the migration period of this species.

\subsection{Ema Celeste, Guyra Nhandu or Celestial Rhea}

The constellation of Celestial Rhea is a constellation of the Tupi Indians from Brazil. The most interesting aspect of this constellation is the fact that this indigenous constellation does not use only the stars to compound its shape. It uses other sky objects, such as the Coalsack Dark Nebula (situated in the Southern Cross, representing the head). To the Tupi, the rhea is related with the myth of the end of the world, because Crux is holding the head of this animal. If the rhea releases itself, it will drink all the water of the world. The Celestial Rhea has a large angular size, encompassing the Crux, the Scorpius, the Centaurus, the Ara, the Musca and the Triangulum Australis.

\subsection{Orion}

Orion is a pre-historic constellation whose registers (engraved in stone) date from 34000 to 29000 B.C. It is known by the asterism Orion's Belt (or The Three Kings) and compounds a quadrilateral structure containing the belt in the center. It has one of the best observable nebulae by naked eye or using small instruments. Because the line of the celestial equator crosses Orion's Belt, it is an excellent mark for the orientation by the equatorial coordinates and for the study of Positional Astronomy. Starting from this constellation, we can identify many others, related both with legends (such as the Canis Major and the Canis Minor and the Taurus) and with mnemonic alignments (such as Lepus and Gemini).

\subsection{Scorpion}

Since the primordial times, the constellation of Scorpion is recognized by many ancient cultures, such as the Babylonians, the Sumerians, the Persians and the Egyptians (the Egyptians represented the Scorpion as a crocodile). It was always associated to bad luck, maybe because the animal itself. Today, with new parameters to divide constellations, the Sun spends fewer day in zodiac than in its neighbour constellation: Ophiucus. In a first moment, the Scorpius was represented with its pincers, but later they were separated to originate the constellation of Balance (Libra). 


\section{Conclusion}

Based on the empirical results, we can verify the point that was originally established. We can conclude that this method is more efficient than the use of direct observation somewhere in the sky. It was also concluded that both methods, if they were used together, have a high performance in the didactic point of view. Using the proposed method to the lay public has an higher efficiency than the conventional method, because the use of phenomena to introduce the astronomy implies a previous knowledge, which is not necessary in this method, since it is based in history and mythology of ancient cultures, a subject that the lay public has a basic concept, just enough to absorb what is being taught. In a local scale, we noticed that the amount of students increased as the time went by. There were always new students that had some kind of relationship (relatives or friends) with another student that belonged to the group, showing the diffusion of the knowledge with the social nucleus where they live.

\section{References}

Azevedo, R. de 1979, No Mundo da Estelândia (São Paulo: Editora do Brasil) de Freitas Mourâo, R. 1981, Atlas Celeste (Rio de Janeiro: Vozes)

de Freitas Mourâo, R. 1995, Manual do Astrônomo (Rio de Janeiro: Jorge Zahar)

Delerue, A. 1970, Rumo às estrelas (Rio de Janeiro: Jorge Zahar)

Lacroux, J. \& Berthier, D. 1988, Lunettes et telescopes - mode d'emploi (Paris: Bordas)

Nicolini, J. 1985, Manual do Astrônomo Amador (São Paulo: Papirus)

Norton, A. P. A. 1954, Star Atlas (Edinburgh: Gall \& Inglis)

Ronan, C. A. 2001, História ilustrada da ciência da Universidade de Cambridge (Rio de Janeiro: Jorge Zahar)

Vehrenberg, H. 1983, Atlas of Deep-Sky Splendors (Cambridge: Cambridge University Press)

Verdet, J. P. 1987, Le Ciel, ordre et désordre (Paris: Gallimard)

Verdet, J. P. 1990, Une historie de l'astronomie (Paris: Editions du Seuil)

Vieira, F. 1999, Identificação do Céu (Rio de Janeiro: Fundação Planetário da Cidade do Rio de Janeiro) 